\title{
Research
}

\section{GPs' views in five European countries of interventions to promote prudent antibiotic use}

\begin{abstract}
Background

A variety of interventions have been developed to promote a more prudent use of antibiotics by implementing clinical guidelines. It is not yet clear which are most acceptable and feasible for implementation across a wide range of contexts. Previous research has been confined mainly to examining views of individual interventions in a national context.
\end{abstract}

\section{Aim}

To explore GPs' views and experiences of strategies to promote a more prudent use of antibiotics, across five countries.

\section{Design and setting}

Qualitative study using thematic and framework analysis in general practices in Belgium

France, Poland, Spain, and the UK.

\section{Method}

Fifty-two semi-structured interviews explored GPs' views and experiences of strategies aimed at promoting a more prudent use of antibiotics. Interviews were carried out in person or over the telephone, transcribed verbatim, and translated into English where necessary for analysis.

\section{Results}

Themes were remarkably consistent across the countries. GPs had a preference for interventions that allowed discussion and comparison with local colleagues, which helped them to identify how their practice could improve. Other popular components of interventions included the use of near-patient tests to reduce diagnostic uncertainty, and the involvement of other health professionals to increase their responsibility for prescribing.

\section{Conclusion}

The study findings could be used to inform future interventions to improve their acceptability to GPs. Consistency in views across countries indicates the potential for development of an intervention that could be implemented on a European scale.

\section{Keywords}

antibacterial agents; attitude of health personnel; quideline adherence; qualitative research; primary health care.

\section{INTRODUCTION}

Antibiotic resistance is recognised as an international health concern due to its potential to increase morbidity and mortality from illnesses that are currently treatable. There is strong evidence that countries with higher prescribing rates have higher rates of antibiotic resistance..$^{1-3}$ GPs are responsible for $80 \%$ of outpatient prescribing, ${ }^{3,4}$ and up to $75 \%$ of these prescriptions can be for respiratory tract infections (RTIs).,5 Much prescribing is unnecessary because infections are selflimiting, and antibiotics make little difference for either upper or lower RTIs. ${ }^{6-8}$ To try to reduce unnecessary prescribing, many countries have introduced guidelines for the management of RTIs.

Qualitative research can be valuable for identifying reasons why GPs differ in how they follow guidelines. ${ }^{9-11}$ Previous research has found that decisions can be based on GPs' desire to maintain good patient relationships, ${ }^{12,13}$ a result of GPs choosing to prioritise patients' immediate perceived demands over long-term issues, ${ }^{14}$ or a result of worries about complications. 13,15,16 Authors concluded that guidelines should include information to help identify patients

S Tonkin-Crine, MSc, senior research assistant P Little, MD, MRCP, FRCGP, professor of primary care research, Aldermoor Health Centre; L Yardley, MSc, PhD, professor of health psychology,

Department of Psychology University of

Southampton, Southampton, UK. S Coenen, MD, $\mathrm{PhD}$, professor of general practice, University of Antwerp, Centre for General Practice, Vaccine \& Infectious Disease Institute (VAXINFECTIO), Belgium. P Fernandez-Vandellos, MPH, research nurse, Hospital Clinic de Barcelona, Applied Research in Respiratory Diseases, Barcelona, Spain. J Krawczyk, MD, FPh, researcher,

Uniwersytet Medyczny w Lodzi, Zaklad Medycyny Rodzinnej i Medycyny Spolecznosci Lokalnych, Lodz, Poland. P Touboul, MD, senior research assistant in general practice and public health Departement de Sante Publique, Hopital de l'Archet 1, Nice, France. T Verheij, MD, PhD, more at risk of complications, should emphasise patients' individual risk from antibiotics, and should emphasise positive aspects of non-antibiotic treatment.

While recommendations are helpful, guidelines are not enough alone to change behaviour. ${ }^{17,18}$ Interventions have been trialled to try to reduce inappropriate prescribing, but it is not clear which are more successful and why. ${ }^{18}$ Intervention design is crucial to ensure guidelines are perceived as feasible by GPs and therefore have the potential to be effective. Qualitative studies investigating GPs attitudes to interventions have only examined attitudes to a specific intervention, ${ }^{19}$ with the exception of a study by Cals et al, who investigated attitudes to two interventions. ${ }^{20}$ No qualitative study has examined GPs opinions on a range of interventions.

It is not clear whether GPs from different countries require different strategies. One study investigating GPs attitudes to guidelines in Norway and Denmark found mainly similar views. ${ }^{21}$ The exception was guidelines that considered the cost of care, which were seen as acceptable by Danish GPs but not by Norwegian GPs who felt that clinical considerations were the only

MRCGP, professor of general practice, University Medical Center Utrecht, Julius Center for Health Sciences and Primary Care, Netherlands.

\section{Address for correspondence}

Miss Sarah Tonkin-Crine, University of Southampton, Primary Medical Care, Aldermoor Health Centre, Aldermoor Close, Southampton, S016 5ST.

E-mail: sktc1007@soton.ac.uk

Submitted: 31 August 2010; Editor's response: 28 September 2010; final acceptance:

17 December 2010.

CBritish Journal of General Practice

This is the full-length article (published online 26 Apr 2011) of an abridged version published in print. Cite this article as: Br J Gen Pract 2011; DOI: 10.3399/bjgp11X572445. 


\section{How this fits in}

Unnecessary prescribing can directly contribute to antibiotic resistance. Although multiple strategies exist to change the prescribing behaviour of GPs, it is not clear which interventions are most acceptable to GPs, and therefore likely to be effective. Previous research has principally focused on GPs' views of individual interventions and on a national basis. This research compares the views of GPs, from several European countries, on multiple strategies, and produces ideas for future interventions. The results suggest that providing opportunities for discussion and comparison between peers, providing near-patient tests for use in consultations, and extending education to patients and other health professionals may improve the acceptability of interventions with GPs and aid implementation. Consistency in GP' views suggests there is potential for producing an intervention that would be suitable for implementation in a variety of European settings.

relevant factor. An important question to consider is whether there are significant variations between less-similar countries in what features of strategies GPs view as acceptable. If there are, there is a need to develop implementation strategies for each country. If not, then an implementation strategy that proves effective in one country can be applied in others.

Studying different countries implies differences in the context in which GPs work, and this incorporates a variety of socioeconomic, cultural, and organisational factors that can influence decision making. Within the current topic, it is therefore important to consider differences between national prescribing rates and national guidelines, to establish what different populations may view as acceptable behaviour. It is also important to consider previous experience with antibiotic interventions to assess whether GPs are familiar with understanding and responding to messages on behaviour change concerning antibiotic prescribing. Overall, in order to assess such potential differences, it would be appropriate to collect a sample that varies in each of these aspects.

This study elicited the views of GPs from five countries, regarding strategies for antibiotic prescribing. It aimed to determine whether there were common features of guidelines and interventions (strategies) that GPs working in very different contexts found desirable and necessary. It also investigated whether there were important contextual differences in GPs views of strategies. The aim was to develop recommendations for developing guidelines and interventions that would be sufficiently broad and flexible to be relevant to a wide range of contexts.

\section{METHOD}

Countries were selected to produce a sample with differences in national prescribing rates: high prescribing in Belgium and France, lower prescribing in the UK, and intermediate prescribing in Poland and Spain. ${ }^{22}$ It was desirable to include GPs with experience of several strategies, and these were more likely to be found in countries with national antibiotic campaigns. France and Belgium represented countries that had had extensive national campaigns, with Spain and the UK having had smaller campaigns, and Poland representing a country with no previous campaigns. ${ }^{23}$ Campaigns most often included guidelines, media messages, and GP seminars.

All participants were GPs. The study aimed to sample high and low prescribers to assess potential differences in attitudes, but this was only possible with certainty in Spain and the UK. British GPS were recruited from high- or low-prescribing surgeries, based on the relevant county average. In Spain, prescribing data were assessed by the percentage of antibiotics prescribed, per GP, compared to the total prescribed for their department. Other countries sampled participants from different locations, to gather GPs with different populations and potentially different prescribing rates. GPs were offered reimbursement to pay for their time to participate in the study. Recruitment continued until researchers were satisfied that no new themes were emerging in interviews.

Six primary care researchers, two of whom were GPs, interviewed participants. Two interviewers were used in France. Interviews were face to face in Poland and Spain, and by telephone in the UK; both methods were used in Belgium and France. 


\section{Box 1. English version of the interview schedule}

\section{Part A: asking about recommendations and guidelines}

What are your views of prescribing antibiotics for respiratory infections?

What are your views of providing guidelines for prescribing antibiotics?

Can you tell me about any guidelines or other forms of advice or recommendations you have received?

For each different form of guidance mentioned/presented ask:

4. What did you think of these recommendations?

5. How did you feel about putting them into practice?

6. How do you think your colleagues felt about the guidelines?

7. Can you describe any situations in which you felt that following the guidelines would be/was helpful?

8. Can you describe any situations in which you felt that following the guidelines would not be/was not helpful?

9. How do you think patients felt about you following these guidelines?

10. How do you think the guidelines might have been improved?

11. What could be done to make it easier to follow the guidelines?

\section{Part B: asking about interventions to help follow guidelines}

1. Can you tell me about any experiences you have had of interventions to help you follow the recommendations?

For each different intervention mentioned and/or for each of the four interventions of interest:

2. What did you think of this intervention?

3. How did you feel about using the intervention/putting it into practice?

4. How do you think your colleagues felt about the intervention?

5. Can you describe any situations in which you felt that following the intervention would be/was helpful?

6. Can you describe any situations in which you felt that following the intervention would not be/was not helpful?

7. How do you think patients felt about you using this intervention?

8. How do you think the intervention might have been improved?

9. What could be done to make it easier to use the intervention? helpful or unhelpful, and whether the strategy could be improved or made easier to follow. Interviews were audiotaped, transcribed verbatim, and anonymised. Spanish, Flemish, and Polish interviews were translated into English by translators in each country. French interviews were translated in the UK. Translations were checked by the interviewer(s) in each country.

Analysis was carried out in the UK, and followed techniques taken from framework and thematic analysis. ${ }^{24,25}$ The use of thematic analysis allowed an inductive approach, which avoided fitting data into pre-existing categories based on either previous research or the researchers preconceptions. A framework approach allowed the large dataset to be handled in systematic stages to aid transparency of results, and allowed charting of data to aid comparisons between countries. Following familiarisation, techniques from thematic analysis were used to develop a thematic framework. One researcher coded initial transcripts, line by line, to produce low-level codes, which were combined to form themes that were checked by two other researchers. NVivo 8 was used to facilitate coding of the data. Analysis occurred alongside data collection, and themes were revised and refined throughout. The remaining data were indexed according to these themes by one researcher, with ongoing checks by another researcher to ensure validity. Charting allowed data to be mapped clearly to aid interpretation and allow direct comparison between countries. Results were discussed within the international research team.

\section{RESULTS}

Fifty-two GPs were interviewed. Interviews ranged from 11 to 69 minutes, with a mean of 33 minutes. Interviews in Belgium and the UK were longer on average, and GPs tended to give more detailed descriptions of their own experiences. Half of the British GPs came from high-prescribing practices, and six Spanish GPs were identified as high prescribers. Participant demographics were similar across all countries (Table 1).

Participants had similar experience, except in Spain where GPs were younger (Table 2). GPs from France and Belgium had fewer partners, which was representative of the typical surgery size in these countries. 


\begin{tabular}{|c|c|c|c|c|c|}
\hline Characteristic & $\begin{array}{l}\text { Belgium } \\
(N=10)\end{array}$ & $\begin{array}{l}\text { France } \\
(N=11)\end{array}$ & $\begin{array}{l}\text { Poland } \\
(N=10)\end{array}$ & $\begin{array}{l}\text { Spain } \\
(N=10)\end{array}$ & $\begin{array}{c}\text { UK } \\
(N=11)\end{array}$ \\
\hline \multicolumn{6}{|l|}{ Age, years } \\
\hline Mean & 45.3 & 49.7 & 42.9 & 38 & 44.5 \\
\hline Range & $29-62$ & $33-58$ & $36-53$ & $29-53$ & $33-56$ \\
\hline \multicolumn{6}{|l|}{ Sex, $n$} \\
\hline Male & 7 & 7 & 6 & 3 & 5 \\
\hline Female & 3 & 4 & 4 & 7 & 6 \\
\hline
\end{tabular}

Spanish GPs came from one city health centre, which was only representative of urban surgeries.

Seven themes were identified, which were consistent across all countries.

\section{Comparison with peers}

The most popular intervention was educational meetings, with GPs showing great interest in their colleagues prescribing. There was a preference for small, interactive events where it was easy to ask questions and hear about others experiences:

We had an opportunity to listen ... and to exchange the information we possessed ... when one heard that his or her colleague was trying not to apply antibiotics and everything was all right ... then one might think that it could be good not to apply antibiotics too.' (Polish, 10)

Many described the aim of meetings was to make peer comparisons and to learn whether their own prescribing could improve and how. Some felt this was useful for GPs whose initial training may have differed more widely from current guidelines. Prescribing feedback, where GPs received data on the number of prescriptions issued in a given period, was another intervention that was praised for its ability to make comparisons. GPs felt it was influential in identifying whether they and their partners were prescribing more antibiotics than average:

I think ranking against other practices is very powerful, you know if you were to list influences of GPS, probably below money would come peer-group comparison.' (British, 5)

\section{Support for non-prescription decisions}

Several GPs reported that interventions that offered practical or financial support had been helpful. Preference for the type of support was split between countries and was dependent on experience. In France

\begin{tabular}{|c|c|c|c|c|c|}
\hline Characteristic & $\begin{array}{l}\text { Belgium } \\
(N=10)\end{array}$ & $\begin{array}{l}\text { France } \\
(N=11)\end{array}$ & $\begin{array}{l}\text { Poland } \\
(N=10)\end{array}$ & $\begin{array}{l}\text { Spain } \\
(N=10)\end{array}$ & $\begin{array}{c}\text { UK } \\
(N=11)\end{array}$ \\
\hline \multicolumn{6}{|l|}{ Years in practice } \\
\hline Mean & 17.5 & 18.8 & 17.4 & 10.4 & 14.8 \\
\hline Range & $2-33$ & $5-30$ & $11-28$ & $2-26$ & $3-30$ \\
\hline \multicolumn{6}{|c|}{ Years in current surgery } \\
\hline Mean & 6.3 & 16.5 & 8.3 & 5.2 & 12.3 \\
\hline Range & $1-33$ & $5-30$ & $3-15$ & $1-8$ & $2-24$ \\
\hline \multicolumn{6}{|c|}{ GPs in current surgery, $n$} \\
\hline Mean & 2.4 & 1.4 & 4.6 & 40a & 7 \\
\hline Range & $2-5$ & $1-4$ & $2-10$ & $\mathrm{NAa}$ & $2-10$ \\
\hline \multicolumn{6}{|c|}{ GPs' surgery location, $n$} \\
\hline Urban & 5 & 4 & 8 & 10 & 6 \\
\hline Rural & 5 & 7 & 2 & 0 & 5 \\
\hline
\end{tabular}


and Poland, GPs favoured near-patient tests, which reduced diagnostic uncertainty while helping to explain non-prescription decisions to patients:

'When there was a mother with her child who had a high temperature and whose tonsils looked ... suspicious, I took a smear in order to do a test and when the result was positive I applied an antibiotic and when it was negative I did not apply it. In this way these tests helped me.' (Polish, 8)

France was the only country where nearpatient tests were widely available; Polish GPs had only experienced using tests through research-funded interventions.

GPs from Spain and the UK supported financial incentives, which they felt gave clinicians more motivation to change:

'The new contract says what financial incentives does, they didn't think general practice would change, they thought no one would achieve the targets and when there was money on the table, the whole organisation, across the country, changed dramatically, almost overnight.' (British, 4)

\section{Lack of support from other health professionals}

GPs were keen for education to be broader. Several felt they were being targeted as a group, while other secondary care professionals had fewer restrictions on their practice. One GP described the ease with which hospital doctors could prescribe and how this impacted on subsequent demand in primary care:

'[Doctors in emergency departments] they do tend to hand out antibiotics like Smarties and then it makes our job a lot more difficult in the surgery to explain to somebody that its probably viral and then hear "but last time doctor they gave me penicillin at the hospital and it sorted it all out because I was much better within the week", and you know they might have been much better within the week without treatment anyway. (British, 10).

Nurse practitioners are also able to prescribe for acute infections in the UK, but GPs did not mention this group and it was unclear whether they felt that education should also be increased for this group.
GPs in Poland and Spain reported the greatest difficulty in prescribing when their patients had already accessed antibiotics prior to a consultation illegally, which was uncommon in other countries. GPs reported that patients managed to obtain antibiotics over the counter and that pharmacists gave drugs to patients on the understanding that a prescription would eventually be obtained from a GP:

'The only thing the MBOs ['Management by Objectives'; a Spanish incentive scheme, to reward primary care practitioners for achieving set targets as determined partly by the government and also by health centres] do is make you argue with the patient ... asking you for a prescription that they have already paid for at the chemist's, and that is not being a doctor, but a policeman.' (Spanish, 7)

\section{Delivering and promoting guidelines}

Most GPs found guidelines helpful, with some reporting that they made them feel 'safer' because they were following recommended practice:

Interviewer: 'And how do you feel applying the advice given in these meetings?'

GP: 'Well ... in general I feel much safer ... you have the feeling that you are carrying on daily life with a much more scientific basis behind it. You feel that apart from your own clinical experience, it is supported by true information.' (Spanish, 3)

GPs from different countries reported receiving different quantities of guidelines, which appeared to correspond with the prevalence of national campaigns in countries. GPs from the UK, France, and Belgium, who had experienced larger, repetitive campaigns, reported being overloaded with information, and those who had no experience of national campaigns or shorter campaigns reported receiving either limited information or none at all:

'Since I started working here I haven't seen any of these guides, I have to go to CAMFIC [Societat Catalana de Medicina Familiar i Comunitària - Catalan Society of Family and Community Medicine] and find out someway that they exist. Here no dissemination about their existence is done.' (Spanish, 3) 


\section{Lack of trust in recommendations}

GPs accepted the content of guidelines and were supportive of efforts to tackle antibiotic resistance, but did not always trust specific recommendations within guidelines. GPs were suspicious about the influence of cost over clinical considerations:

'I've been in on meetings, being an exprescribing lead, where it's so heavily biased in the financial direction that I wondered if clinical judgment and clinical practice were being thought about at all.' (British, 7)

They also reported conflicting information between different sources of advice:

'Well, some things are well done, and some others are much too strict. But, well, especially with the guides from the ICS [Institut Català de la Salut - the Catalan Institute of Health is the main public provider of health services in Catalonia, Spain], what happens is that they do not coincide with the guides published by the Spanish Society of Pneumology, and these kinds of contradictions might cause problems.' (Spanish, 10)

A few GPs simply did not believe in specific recommendations, despite accompanying research evidence, because it seemed contradictory to their own experience:

'I know bronchitis is viral. Still, most of the time I see, in the usual clinical practice, that giving antibiotics shortens the duration of bronchitis partly or to a large extent, which makes me doubt whether in the viral process of bronchitis there is also some bacteria involved.' (Spanish, 7)

Lastly, some GPs worried about whether advice was independent:

'I don't think that you can find 100\% objective information ... because those pharmaceutical companies will always be involved in the funding at some level. (Belgian, 7)

All GPs stated that providing a clear evidence base strengthened their trust in recommendations.

\section{Barriers to following recommendations} Almost all GPs felt their practice already matched guidelines, with some describing certain recommendations within guidelines as 'obvious':

'Those [recommendations] are all the ones we would do anyway so I don't know if I read those guidelines or not but that's standard practice isn't it; if you ask me, that's being a bit patronising because that's what we do anyway.' (British, 10)

Despite this, some GPs seemed to focus on changing antibiotic type, from a secondline antibiotic to a first-choice drug, rather than reducing prescribing overall, suggesting that they may not have understood the message of reduced prescribing. This included GPs who had received a lot of information on recommendations:

II mean that's the bottom line really to get the prescribing away from sort of secondline prescribing.' (British, 1)

GPs described two situations where guidelines were difficult to follow; first where they feared negative clinical consequences of not prescribing:

I mean sometimes although an infection seems to be simple, a patient is feeling terrible or he has a high temperature, so one thinks that a stronger antibiotic should be used even though the guideline says something different. And then I don't feel quite comfortable with [following the guideline], because I can imagine that he will come back ... and he won't feel better at all.' (Polish, 3)

Secondly, where there was situational pressure to prescribe:

'When you go home from these meetings and you think you know I'm going to be good, I'm not going to do this, I'm not going to do that ... but you know sometimes, last patient on a Friday night ...' (British, 11)

GPs justified prescribing in some inappropriate situations, based on the observation that 'guidelines are guidelines', implying that recommendations were not strict rules but suggestions for practice. 


\section{Education for patients}

All GPs reported having patients who had expectations for antibiotics, and felt education would help reduce these expectations. Some worried about not prescribing, as they felt patients would go to another doctor:

'There is a group of mothers that go to another doctor if they learn that I am not going to prescribe an antibiotic.' (Polish, 10)

Some GPs stated that they used guidelines to support their decisions and to educate patients:

'I tell them that that's the way I do it and in addition, when they are a little insistent, I tell them "there are French recommendations which are called that, that, and that ...", and I show them, on the internet, you know, I show them, they read them, they see them, and like that, they understand very well. (French, 11)

Others felt that patients' trust in them helped them to accept decisions, and that patients were unconcerned about guidelines:

I think that if my patients are satisfied with me, they would continue being satisfied whether I used these guides or not ... they trust the professional, not the guideline. (Spanish, 3)

Educational materials for patients and media campaigns for the public were generally supported, and GPs mentioned that they often helped to reduce demand for antibiotics:

'Well we had a campaign about 5 to 10 years ago didn't we, the same sort of thing, a lot of it on television and it made it easier because patients said "oh yes l've heard of that". It works, or it did work. It made it easier in the consultations. I think it made it easier not to give antibiotics.' (British, 4)

A few GPs from all countries admitted that prescribing was often quicker and easier even when not required, and that this option was sometimes preferred over explaining decisions to patients:

'Because it seems that the days where there are lots of people, I prescribe a lot more ... because it's easier, you get rid of the person quickly by giving them the medicine and then they go. If you spend a quarter of an hour talking to the patient, and well, you can't see 50 in a day, it's not possible. (French, 7)

\section{DISCUSSION}

\section{Summary}

This was the first qualitative study to explore GP attitudes to multiple strategies across different European countries. The key finding was that, despite differences in context, GPs held broadly similar views and preferences for strategy components. These findings suggest it may be feasible to develop an implementation strategy that will be widely applicable. Where differences arose, they were a result of differences in either GPs' experience of strategies, or the availability of antibiotics in different countries, both of which are explained below.

The study results indicate what elements an implementation strategy should include in order to maximise acceptability with GPS. One new finding to emerge was that GPs found educational meetings acceptable and feasible as an intervention. GPs placed great importance on discussion and comparison with their peers, and these elements were favoured because they gave relevant, local information, which GPs prioritised over general, national data. Receiving information from local colleagues and making direct comparisons reassured GPs that more prudent prescribing was possible. A second new finding concerned the popularity of near-patient tests as a practical tool, as participants felt tests aided diagnosis and could be used to support explanations to patients.

GPs reported that guidelines helped them feel 'safer', as they were following recommended practice, suggesting how guidance may be made more attractive. Lastly, it was clear that GPs felt that guidelines and interventions needed to target other health professionals, because they felt antibiotics were given too frequently to patients, which undermined their attempts to change. Education delivered with prescribing feedback would probably be a suitable solution for this.

Three main differences emerged between comments from participants in different 
countries, all relating to differences in their experiences and local context. First, participants had varying experience of receiving guidelines on antibiotic prescribing, which affected their enthusiasm for receiving further advice; the desire for guidance was greater among those who had received little. Secondly, GPs experienced different barriers within their health system in changing their prescribing behaviours, with GPs from Spain and Poland reporting that patients could access antibiotics prior to a consultation because dispensing laws were not enforced. Thirdly, participants differed in what they felt could improve their prescribing, with a split between near-patient tests and financial incentives. Incentives were directly asked about in interviews, and those GPs who had experience with them, in Spain and the UK, felt they were helpful. However, when asked about incentives, GPs without experience tended to disapprove of the idea and did not believe it would change their practice. Although this may be true, comments may also reflect socially desirable responses, with GPs not wishing to appear to be, or to believe that they could be, influenced by financial factors. Near-patient tests were only mentioned by GPs with experience of using tests who found them helpful. The use of near-patient tests incurs costs in primary care, which is likely to be a concern to GPs and is therefore an important factor to consider when considering implementation.

\section{Strengths and limitations}

Voluntary participation meant that samples may not have represented typical views for each country. Sampling to include a diverse range of prescribers was not always possible; however, it was encouraging to see that attitudes between high and low prescribers in the UK and Spain did not differ, indicating that preferences for strategy elements may be similar despite different rates of prescribing.

The method led to other issues that could not be avoided. In one country, recruitment difficulties resulted in all GPs coming from the same health centre, which meant attitudes between the participants may have been more similar, as a result of working in the same setting. Interviews were carried out in person or by telephone, and two of the five interviewers were GPs, both of which may have influenced responses, although there was no evidence in the data to suggest this in either case. Lastly, the translation of interviews may have resulted in lost or misrepresented data; therefore, translations were checked by interviewers to confirm that data were representative of the original text.

There is always a risk that participants will express socially desirable, rather than genuine, attitudes. To try to elicit genuine opinions, interviewers presented themselves as independent researchers and reassured GPs of their desire to understand, not judge, their decisionmaking processes. Since several GPs were happy to state their negative opinions of strategies, the researchers were confident that the group were able to express their true feelings.

\section{Comparison with existing literature}

The study findings suggest that there are multiple strategy components that GPs find attractive, indicating that a multifaceted intervention may be appropriate; this is in line with a previous review of the effectiveness of interventions to reduce prescribing. ${ }^{18}$ The two new findings emerging from the research support previous quantitative work by giving some indication as to why interventions comprising group discussions, feedback, and near-patient tests are effective at improving antibiotic use in primary care. ${ }^{5,26,27}$

GPs' preference for peer discussion and comparison supported the idea that local information was preferred over national recommendations; this is consistent with previous work suggesting that a consensus on practice can be interpreted to be context specific. ${ }^{28}$ The remaining themes emerging from the data were consistent with previous qualitative research. Clinicians found it difficult to trust guidelines; this is consistent with research on GP attitudes to guidelines in general, and highlighted the need for a clear and explicit evidence base behind recommendations. ${ }^{10,11,21} \mathrm{~A}$ further barrier to following recommendations was also the concern clinicians reported about the potential risks of not prescribing, which relates to other research suggesting that interventions need to help GPs identify those patients who are more at risk. ${ }^{14-16}$ This concern did not appear to differ between participants across countries. Lastly, clinicians discussed the pressure of patient 
expectations and the desire to respond to these, to either make the patient happier or the consultation easier. Although participants did not report differences in the extent of patient demand, GPs from the UK and Belgium claimed that demand was more prevalent in patients originally from other European or African countries, which has also been indicated in research in other countries. ${ }^{29}$ The influence of demand is consistent with previous studies that have suggested educational materials for patients, ${ }^{30}$ although these may be most effective if tailored to highlight the risks of antibiotics for individuals. ${ }^{12,15}$

\section{Implications for practice and research}

The consistency between views of participants from different countries enables formulation of ideas to improve strategies that should be relevant for all contexts involved. The study results suggested that interventions may benefit from allowing peer discussion and comparison between GPs in a local area, and that providing practical equipment such as near-patient tests may help increase confidence in appropriate non-prescription of antibiotics. Promoting guidelines as a way to achieve 'safer' practice may also make advice more attractive, and, in addition, education should be tailored to patients and other health professionals. Strategies such as financial incentives appear to be relevant only to some contexts, which suggests slight tailoring to specific settings may be necessary if such items are used.

Future research should incorporate such aspects into a multifaceted intervention, to be tested in an EU clinical trial, to assess the effectiveness and potential barriers of such an approach. Most of the suggestions do not require significant input of resources, but some, such as peer discussions, do have significant resource implications; therefore, not only would they have to be shown to be efficient, but they might also require financial incentives to encourage uptake. 


\section{REFERENCES}

1. Costelloe C, Metcalfe C, Lovering A, et al. Effect of antibiotic prescribing in primary care on antimicrobial resistance in individual patients: systematic review and meta-analysis. BMJ 2010; 340: c2096.

2. Malhotra-Kumar S, Lammens C, Coenen S, et al. Effect of azithromycin and clarithromycin therapy on pharyngeal carriage of macrolide-resistant streptococci in healthy volunteers: a randomised, double-blind, placebocontrolled study. Lancet 2007; 369(9560): 482-490.

3. Goossens H, Ferech M, Stichele RV, et al. Outpatient antibiotic use in Europe and association with resistance: a cross-national database study. Lancet 2005; 365(9459): 579-587.

4. Wise R, Hart T, Cars O, Streulens M, et al. Antimicrobial resistance. Is a major threat to public health. BMJ 1998; 317(7159): 609-610.

5. Welschen I, Kuyvenhoven MM, Hoes AW, Verheij TJM. Effectiveness of a multiple intervention to reduce antibiotic prescribing for respiratory tract symptoms in primary care: randomised controlled trial. BMJ 2004; 329(7463): 431-433

6. Little P. Rumsby K, Kelly J, et al. Information leaflet and antibiotic prescribing strategies for acute lower respiratory tract infection - a randomized controlled trial. J Am Med Assoc 2005; 293(24): 3029-3035.

7. Del Mar CB, Glasziou PP, Spinks AB. Antibiotics for sore throat. Cochrane Database Syst Rev 2006; (4): CD000023.

8. Smucny J, Fahey T, Becker L, Glazier R. Antibiotics for acute bronchitis. Cochrane Database Syst Rev 2004; (4): CD000245.

9. Rashidian A, Eccles MP, Russell I. Falling on stony ground? A qualitative study of implementation of clinical guidelines' prescribing recommendations in primary care. Health Policy 2008; 85(2): 148-161.

10. Carlsen B, Glenton C, Pope C. Thou shalt versus thou shalt not: a metasynthesis of GPs' attitudes to clinical practice guidelines. Br J Gen Pract 2007; 57(545): 971-978.

11. Carlsen B, Norheim OF. 'What lies beneath it all?' - an interview study of GPs' attitudes to the use of guidelines. BMC Health Serv Res 2008; 8: 218.

12. Butler CC, Rollnick S, Pill R, et al. Understanding the culture of prescribing: qualitative study of general practitioners' and patients' perceptions of antibiotics for sore throats. BMJ 1998; 317(7159): 637-642.

13. Coenen S, Van Royen P, Vermeire E, et al. Antibiotics for coughing in general practice: a qualitative decision analysis. Fam Pract 2000; 17(5): 380-385.

14. Wood F, Simpson S, Butler CC. Socially responsible antibiotic choices in primary care: a qualitative study of GP' decisions to prescribe broadspectrum and fluroquinolone antibiotics. Fam Pract 2007; 24(5): 427-434.

15. Kumar S, Little P, Britten N. Why do general practitioners prescribe antibiotics for sore throat? Grounded theory interview study. BMJ 2003. 326(7381): 138-141.
16. Simpson SA, Wood F, Butler CC. General practitioners perceptions of antimicrobial resistance: a qualitative study. J Antimicrob Chemother 2007; 59(2): 292-296

17. Feder $G$, Eccles $M$, Grol $R$, et al. Clinical guidelines: using clinical guidelines. BMJ 1999; 318(7185): 728-730.

18. Arnold SR, Straus SE. Interventions to improve antibiotic prescribing practices in ambulatory care. Cochrane Database Syst Rev 2005; (4): CD003539.

19. Watkins C, Timm A, Gooberman-Hill R, et al. Factors affecting feasibility and acceptability of a practice-based educational intervention to support evidencebased prescribing: a qualitative study. Fam Pract 2004; 21(6): 661-669.

20. Cals JWL, Butler CC, Dinant GJ. 'Experience talks': physician prioritisation of contrasting interventions to optimise management of acute cough in general practice. Implement Sci 2009; 4: 57

21. Carlsen B, Kjellberg PK. Guidelines; from foe to friend? Comparative interviews with GPs in Norway and Denmark. BMC Health Serv Res 2010; 10:

22. Muller A, Coenen S, Monnet DL, Goossens H. European Surveillance of Antimicrobial Consumption (ESAC): outpatient antibiotic use in Europe, 1998-2005. Euro Surveill 2007: 12(10): E0710111.

23. Huttner B, Goossens H, Verheii T, et al. Characteristics and outcomes of public campaigns aimed at improving the use of antibiotics in outpatients in high-income countries. Lancet Infect Dis 2010; 10(1): 17-31.

24. Braun V, Clarke V. Using thematic analysis in psychology. Qual Res Psychol 2006; 3: 77-101.

25. Ritchie J, Spencer L. Qualitative data analysis for applied policy research. In: Bryman A, Burgess R (eds). Analysing qualitative data. London: Routledge, 1993; 173-194.

26. Cals JWL, Schot MJC, de Jong SAM, et al. Point-of-care C-reactive protein testing and antibiotic prescribing for respiratory tract infections: a randomized controlled trial. Ann Fam Med 2010; 8(2): 124-133.

27. Llor C, Cots JM, Lopez-Valcarcel BG, et al. Effect of two interventions on reducing antibiotic prescription in pharyngitis in primary care. J Antimicrob Chemother 2011; 66(1): 210-215.

28. Fairhurst K, Huby G. From trial data to practical knowledge: qualitative study of how general practitioners have accessed and used evidence about statin drugs in their management of hypercholesterolaemia. BMJ 1998; 317(7166): 1130-1134.

29. Hogenhuis CC, Grigoryan L, Numans MM, Verheii TJ. Differences in antibiotic treatment and utilization of diagnostic tests in Dutch primary care between natives and non-western immigrants. Eur J Gen Pract 2010; 16(3): 143-147.

30. Butler CC, Rollnick S, Kinnersley P, et al. Reducing antibiotics for respiratory tract symptoms in primary care: consolidating 'why' and considering 'how'. $\mathrm{Br}$ J Gen Pract 1998; 48(437): 1865-1870. 